\title{
Can phenotypic variability of polygenic traits yield information regarding selection?
}

\author{
G. S. Mani
}

Physics Department, University of Manchester, Manchester M139PL, U.K.

The relation between phenotypic variability and heterozygosity for a polyallelic polygenic trait is obtained using computer simulation. It is shown that unlike the di-allelic case, the variance of phenotypic score of the trait can increase or decrease depending on the allele frequency distribution. The relevance of this to selection is discussed.

\section{INTRODUCTION}

In a recent paper Chakraborty (1987) argues that the increased frequency of modal phenotypes in highly heterozygous individuals in a population and the consequent decrease in phenotypic variability can be explained solely on the basis of the additivity of allelic effects of a polygenic trait. It is not necessary to invoke any selective hypothesis to explain this feature. The correlation of phenotypic variability with the individual's heterozygosity has been theoretically studied by Chakraborty and Ryman (1983) where they showed that the phenotypic variability is least for individuals who are heterozygous for most loci. Using this result, Chakraborty (1987) asserts that "polygenic traits with additive allelic effects always produce a negative correlation between heterozygosity (as determined by number of heterozygous loci) and phenotypic variance, and for this to happen there is no need to invoke a selection hypothesis for the traits or for the underlying loci".

The results of Chakraborty (1987) mentioned above were based on a di-allelic multi-loci system. The purpose of this note is to demonstrate that the above assertion of Chakraborty is not valid if one goes to a poly allelic polygenic system and that in general it is possible to ascertain selective effects from the correlation between polygenic variability and the number of heterozygous loci in an individual.

\section{DESCRIPTION OF THE METHOD OF SIMULATION}

The 60-bit word of the CDC7600 computer was divided into 10 6-bit sections corresponding to 10 loci. Thus at each locus a maximum of $2^{6}$ alleles could be maintained. The population size was taken to be 5000 corresponding to 10000 words (or gametes). All loci are assumed to have the same number of alleles, say $k$, and the allelic frequencies $p_{i}, i=1, \ldots, k$, are further assumed to be the same at all loci. The $k$ alleles at each locus are designated by $1,2, \ldots, k$. The allele at any locus of any individual in the population was selected by choosing a random number from a uniform distribution in the range $[0,1]$ and comparing it with the cumulative allele frequencies. Having thus obtained a random distribution of alleles for the population constrained by the given set of allelic frequencies, the number of heterozygous loci and the allele present at each locus for any individual was obtained using a COMPASS routine for bit-wise manipulation.

The character space spanned between the values 0 and 20 . This range was used to enable comparison with the results of Chakraborty (1987). Thus for a $k$ allele, $n$ locus system, the $j$ th allele $(1 \leqslant j \leqslant k)$ contributes a value

$$
\Delta X=\left(\frac{j-1}{k-1}\right) \times\left(\frac{10}{n}\right)
$$


to the character. The alleles at all $n$ loci of an individual contribute additively to the magnitude of the individual's trait. All results were obtained with 100 independent replicates.

\section{RESULTS}

The relationship of the number of heterozygous loci with the mean and with the standard deviation of the phenotypic score of a polygenic trait for the case of 2 and 3 alleles at each locus is shown in fig. 1 . In the case of 2 alleles, the results are the same as quoted by Chakraborty (1987). In the case of 3 alleles the following points emerge:

(a) Unlike the di-allelic case, the mean of the phenotypic score for various sets of allelic frequencies do not converge to a single point with increasing value of the number of heterozygous loci.

(b) The standard deviation is not always a decreasing function of the number of heterozygous loci. In fact when the allele frequencies are such that one allele has a high frequency while all the others are at a very low frequency, the variance of the phenotypic score would

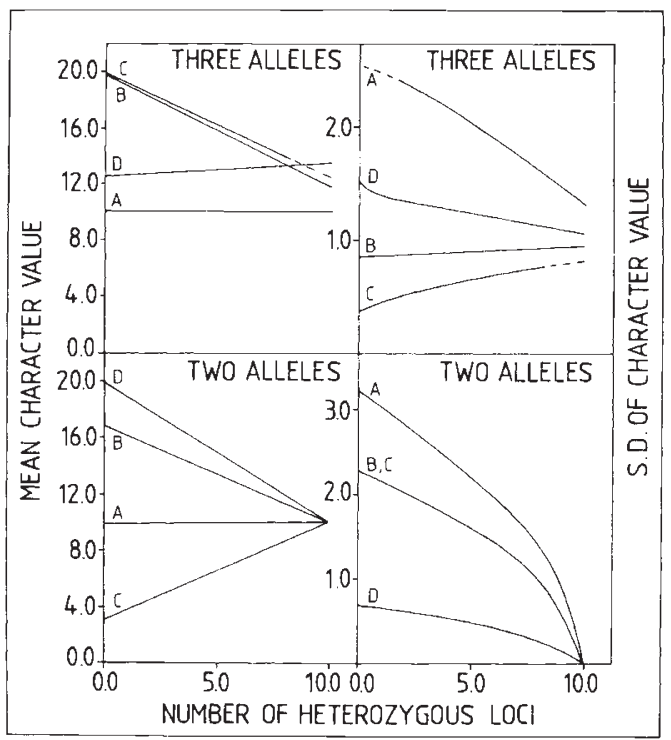

Figure 1 Relationship of the number of heterozygous loci with the mean and standard deviation (S.D.) of the phenotypic score of a polygenic trait for a di- and tri- allelic systems. The various curves in both cases correspond to different sets of allelic (frequencies and are as follows:

$$
\begin{aligned}
2 \text { alleles } & A:\{0.5,0.5\} ; B:\{0 \cdot 3,0.7\} \\
& C:\{0.7,0.3\} ; D:\{0 \cdot 1,0.9\}
\end{aligned}
$$

3 alleles $\mathrm{A}:\left\{\frac{1}{3}, \frac{1}{3}, \frac{1}{3}\right\} ; \mathrm{B}:\{0 \cdot 1,0 \cdot 1,0 \cdot 8\}$ C: $\{0.05,0.05,0.90\} ; D:\{0 \cdot 05,0.60,0.35\}$ increase with heterozygosity. If, on the other hand, the frequencies of all the alleles are equal to $1 / k$, the variance would be a decreasing function of heterozygosity. All intermediate cases would either decrease or increase depending upon whether their allele frequencies are closer to the one or the other of the above two cases, with much lower value of slopes.

These points have been checked for cases up to 6 alleles. The results for 4 alleles at each locus is shown in fig. 2.

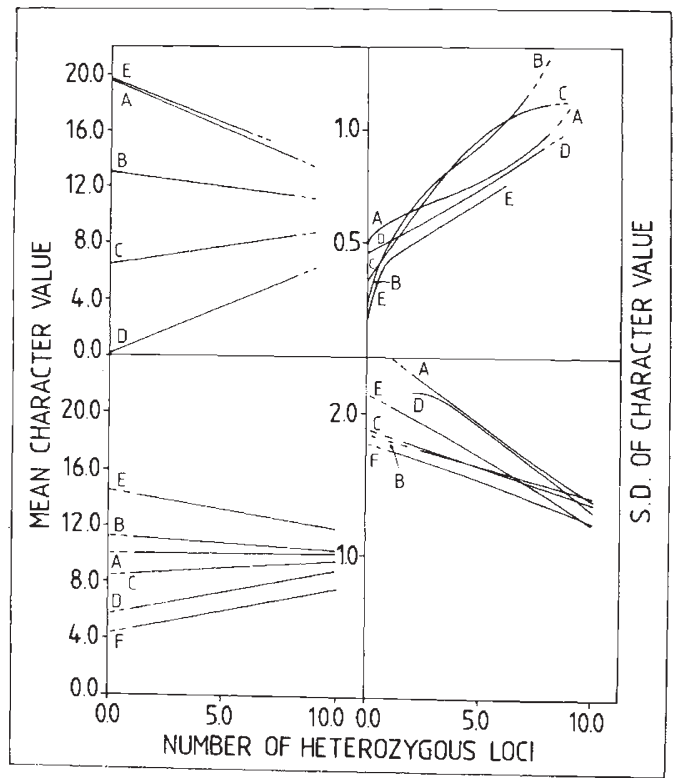

Figure 2 Relationship of the number of heterozygous loci with the mean and standard deviation (S.D.) of the phenotypic score of a polygenic trait for a 4-allelic system. The various curves correspond to different sets of allelic frequencies and are as follows:

Top Curve $A:\{0.05,0.05,0 \cdot 05,0.85\}$

$B:\{0.05,0.05,0.85,0.05\}$

$C:\{0 \cdot 05,0 \cdot 85,0 \cdot 05,0.05\}$

$D:\{0.85,0.05,0.05,0.05\}$

$E:\{0.02,0.02,0.02,0.90\}$

Bottom Curve $A:\left\{\frac{1}{4}, \frac{1}{4}, \frac{1}{4}, \frac{1}{4}\right\} ; B:\{0 \cdot 2,0 \cdot 2,0 \cdot 4,0 \cdot 2\}$

$C:\{0 \cdot 2,0 \cdot 4,0 \cdot 2,0 \cdot 2\} ; D:\{0 \cdot 4,0 \cdot 2,0 \cdot 2,0 \cdot 2\}$

$E:\{0 \cdot 1,0 \cdot 3,0 \cdot 2,0 \cdot 4\} ; F:\{0 \cdot 4,0 \cdot 3,0 \cdot 2,0 \cdot 1\}$

Regions with small sample sizes yielding large uncertainty are denoted by broken lines.

\section{DISCUSSION}

The allelic frequency distribution in the neutral model is predicted to be U-shaped with one allele having a high frequency and all the other alleles being at a very low frequency. Examples of such 
distribution have been given by Chakraborty et al. (1980). On the other hand selective models would predict allele frequencies with most of the alleles close to the value of the mean frequency. In the case of the overdominant model of selection $\mathrm{Li}$ (1978) has shown that the frequency distribution is either bell-shaped or W-shaped. Thus for the neutral model with more than two alleles at each locus, one would expect the phenotypic variance to increase with increasing number of heterozygous loci. On the other hand selective models (including overdominant models) would predict a decrease in the variance with increasing value of heterozygosity. This then provides, at least in theory, a method of ascertaining if selective effects are operating in the population under study. This conclusion no doubt depends on how robust the additive assumption is and on whether environmental effects can be assumed to be small.
Acknowledgement I am grateful to Laurence Cook and Bryan Clarke for many useful discussions.

\section{REFERENCES}

CHAKRABORTY, R., FUERST, P. A. AND NEI, M. 1980. Statistical studies on protein polymorphism in natural populations. III. Distribution of allele frequencies and the number of alleles per locus. Genetics, 94, 1039-1063.

CHAKRABORTY, R., AND BYMANM, N. 1983. Relationship between mean and variance of genotypic values with heterozygosity per individual in a natural population. Genetics, 103, 149-152.

CHAKRABORTY, R. 1987. Biochemical heterozygosity and phenotypic variability of polygenic traits. Heredity, 59, 19-28.

LI, W-H. 1978. Maintenance of genetic variability under the joint effect of mutation, selection and random drift. Genetics, 90, 349-382. 